\title{
Faulted Phase Selection Based on Wavelet Analysis of Traveling Waves
}

\author{
V. S. Kale, S. R. Bhide, and P. P. Bedekar
}

\begin{abstract}
Accurate faulted phase selection is critical to avoid tripping of the incorrect phase or unnecessary three phase tripping. Moreover, an associated requirement of faulted phase selectors is high-speed operation, since the selection process must be completed in the immediate post-fault period before breaker opening. This paper presents a novel scheme for selecting a faulted phase on transmission line based on the traveling wave theory, meeting both the requirements of accuracy and speed. The proposed approach uses the mathematical tool of wavelet modulus maxima to solve the problem. MATLAB/ Simulink software was used to test the proposed faulted phase selection approach. Various fault conditions were simulated by varying fault type, fault resistance, fault location and fault inception angle, on a given power system model. The simulation results demonstrate the validity of the proposed approach of faulted phase selection.
\end{abstract}

Index Terms-Faulted phase selection, traveling wave, wavelet modulus maxima, transmission line.

\section{INTRODUCTION}

Statistically, about $80 \%$ of the faults on the transmission lines are transient in nature. These faults can disappear by themselves, if the supply is briefly interrupted and the arc path is allowed to de-ionize. Hence, auto-reclosing is attempted for the purpose of restoring transmission line to service subsequent to tripping of their associated circuit breaker due to faults [1]. Single pole auto-reclosure has long been recognized as an effective means of improving system security and reliability. Its benefits are particularly apparent in applications where economic and stability considerations preclude the use of three phase auto reclosure. Accurate faulted phase selection is required for implementation of such a scheme [2].

Due to the limitations of the conventional methods, wide variety of faulted phase selection algorithms have been developed and proposed over the years to select the faulted phase(s). Artificial intelligence (AI) techniques such as artificial neural network [3], fuzzy logic [4] and support vector machine [5] had been applied to the problem of faulted phase selection. Mathematical technique such as wavelet transform was used to select the faulted phase [6]. Also

Manuscript received September 30, 2010; revised May 2, 2011.

V.S. Kale is with the Department of Electrical Engineering, Visvesvaraya National Institute of Technology, Nagpur, INDIA (phone: +91-712-2801137; e-mail: tovijay_kale@rediffmail.com).

S. R. Bhide is with the Department of Electrical Engineering, Visvesvaraya National Institute of Technology, Nagpur, INDIA (e-mail: srbhide@yahoo.com).

P. P. Bedekar is with the Department of Electrical Engineering, Visvesvaraya National Institute of Technology, Nagpur, INDIA (e-mail: bedekar_pp@rediffmail.com). combination of $\mathrm{AI}$ and non-AI techniques was used to identify the faulted phase(s) as in [7] and [8].

Ref. [9] proposed faulted phase selection using wavelet transform based on the spectral energies of traveling waves. A fault classification scheme based on spectrum characteristics of traveling wave components obtained using FFT was given in [10]. Modal components of characteristics impedances were considered as fuzzy variables for identifying single line to ground and phase to phase faults [11]. Selection of faulted phase based on the wavelet analysis of modal components of traveling waves was presented in [12].

However, the traveling wave based algorithms proposed were not supported by the results taking into account the effect of wide variation of uncontrolled fault parameters like fault inception angle and fault impedance. This paper presents a novel algorithm utilizing the fault parameters which are based on characteristics of wavelet modulus maxima of modal components of fault induced traveling waves. MATLAB/Simulink technical computing platform was used for offline simulation of wide range of power system fault conditions. It is shown that the proposed algorithm implements a high speed faulted phase selection scheme which operates correctly in variety of situations.

\section{WAVELET TRANSFORM}

The wavelet transform (WT) is a powerful mathematical tool widely used for digital signal processing. WT is useful for the analysis of non-stationary signals such as those associated with faults or switching operations. It has the ability to analyze a localized area of a signal and reveal transitory aspects of data like drift, trends, and abrupt changes. Thus, WT is useful in detecting the onset of a fault and has been widely applied in the field of protective relaying, including high impedance fault detection, faulted phase identification, traveling wave fault location and transformer protection [13].

The function $\psi(\mathrm{t})$ is the base wavelet, if it satisfies the finite energy condition

$$
\int_{-\infty}^{+\infty}|\psi(t)|^{2} d t<\infty
$$

The function family $\psi_{\mathrm{s}, \mathrm{b}}(\mathrm{t})$ generated through dilation parameter ' $s$ ' and translation parameter ' $b$ ' is defined as

$$
\psi_{\mathrm{s}, \mathrm{b}}(\mathrm{t})=\frac{1}{\sqrt{\mathrm{s}}} \psi\left(\frac{\mathrm{t}-\mathrm{b}}{\mathrm{s}}\right), s, b \in R, s \neq 0
$$

where $R$ is a set of real numbers. The wavelet transform of any function $x(t)$ is defined as 


$$
\mathrm{W}_{\mathrm{f}}(\mathrm{s}, \mathrm{b})=\frac{1}{\sqrt{\mathrm{s}}} \int_{-\infty}^{\infty} x(t) \psi^{*}\left(\frac{\mathrm{t}-\mathrm{b}}{\mathrm{s}}\right) d t
$$

$\psi^{*}\left(\frac{\mathrm{t}-\mathrm{b}}{\mathrm{s}}\right)$ is a conjugate of wavelet function $\psi\left(\frac{\mathrm{t}-\mathrm{b}}{\mathrm{s}}\right)$

Wavelet transform $\mathrm{W}_{\mathrm{f}}(\mathrm{s}, \mathrm{b})$ depends on scale factor and translation factor. Through variation of scale factor, the wavelet transform can be applied to high frequency components where short time intervals are necessary. Therefore, it is a suitable approach to analyze the traveling waves. If $s=\frac{1}{2^{j}}(j \in Z, Z$ is a set of integers $)$ and $\mathrm{b} \in \mathrm{R}$ ( $\mathrm{R}$ is a set of real numbers), then it is dyadic wavelet transform. It is translation invariant and hence used in signal edge detection.

Wavelet Modulus Maxima (WMM) of wavelet transform are the local maxima of wavelet transform satisfying the following condition:

$$
\left|W_{m} x(t)\right| \leq A s^{\alpha}
$$

where, $W_{m} x(t)$ is the WMM of signal $x(t)$, A is constant, and $\alpha$ is the Lipschitz exponent.

Modulus maxima represent the singularity of step signal. The polarity of WMM is identical to polarity of sudden change of the signal and its magnitude depends on the amplitude and gradient of the sudden change of the signal. In this paper, WaveLab is used to obtain WMM. WaveLab which is available from Stanford University can be used as an alternative to the MATLAB wavelet toolbox.

\section{TRAVELING WAVE THEORY}

A fault on the transmission line sets up traveling waves which propagate from the fault point towards the line terminals at speeds close to that of light. Three-phase lines have significant electromagnetic coupling between conductors. As a result, during traveling wave conditions, the self and mutual surge impedances affect their behavior and 'single' surge impedance valid during different faults cannot be defined. In numerical relaying applications, this needs matrix manipulations which require more computational time to compute suitable relaying signals. Hence, it is necessary to transform a coupled three phase system to a decoupled systems having surge impedance terms independent of fault type. By means of modal decomposition, the coupled voltages and currents are decomposed into a new set of modal voltages and currents and each transformed signal can be treated independently in a manner similar to that of single-phase line. The relationship between three phase voltage and current signals and their respective modal components is given by

$$
\begin{aligned}
& {\left[e_{p}(t)\right]=[S]\left[e^{m}(t)\right]} \\
& {\left[i_{p}(t)\right]=[Q]\left[i^{m}(t)\right]}
\end{aligned}
$$

where $[S]$ and $[Q]$ are the voltage and current modal transformation matrices and are equal to each other for transposed lines. [ $\left.e^{m}(t)\right]$ and $\left[i^{m}(t)\right]$ are the modal voltage and current matrices. The elements of transposed matrix can be determined using matrix function theory and the properties of eigen values and eigen vectors. In protection applications, three of the widely used constant modal transformation matrices for perfectly transposed lines are the Clarke, Wedepohl, and Karrenbauer transformation. In the present work, Karrenbauer transformation matrix is used.

Phase currents can be viewed as being made up of $i^{(0)}, i^{(1)}$ and $i^{(2)}$ i.e. mode- 0 , mode- 1 and mode- 2 components. Each of these components represents a mode of the propagation equation. The mode- 0 component has a characteristics impedance and velocity of propagation that are distinct from those of modes-1 and mode- 2 components. Using Karrenbauer transformation, we can write

$$
\left[\begin{array}{l}
i^{(0)} \\
i^{(1)} \\
i^{(2)}
\end{array}\right]=\frac{1}{3}\left[\begin{array}{ccc}
1 & 1 & 1 \\
1 & -1 & 0 \\
1 & 0 & -1
\end{array}\right]\left[\begin{array}{l}
i_{a} \\
i_{b} \\
i_{c}
\end{array}\right]
$$

From (2), we can express $i^{(1)}$ and $i^{(2)}$ as

$$
\begin{aligned}
i^{(1)} & =\frac{1}{3}\left(i_{a}-i_{b}\right) \\
i^{(2)} & =\frac{1}{3}\left(i_{a}-i_{c}\right)
\end{aligned}
$$

As can be seen from (3) and (4), mode-1 component of the current is related to currents of phases ' $a$ ' and ' $b$ ' and mode- 2 component of current is related to currents of phases ' $a$ ' and 'c'. Similarly we can write mode- 3 component of current which will be related to currents of phases ' $b$ ' and ' $c$ ' as given in (5).

$$
i^{(3)}=\frac{1}{3}\left(i_{b}-i_{c}\right)
$$

\section{A. Fault parameterization}

The analysis for various types of fault can be performed to obtain the conditions which are unique to the individual fault. Consider AG fault which is defined by the boundary conditions: $i_{a}=i_{b}=0$ and $v_{a}=0$. This condition when substituted in the definition of Karrenbauer components leads to $i^{(0)}=i^{(1)}=i^{(2)}=\frac{1}{3} i_{a}$ and $i^{(3)}=0$.

Consider BC fault defined by the boundary conditions: $i_{a}=0 ; i_{b}=-i_{c}$ and $v_{b}=v_{c}$. This condition when substituted in the definition of Karrenbauer components leads to $i^{(0)}=0 ; i^{(1)}=-\frac{1}{3} i_{b} ; i^{(2)}=\frac{1}{3} i_{b}$ and $i^{(3)}=\frac{2}{3} i_{b}$.

Now consider BCG fault which is defined by the boundary conditions: $i_{a}=0$; and $v_{b}=v_{c}=0$. This condition when substituted in the definition of Karrenbauer components leads to $i^{(0)}=\frac{1}{3}\left(i_{b}+i_{c}\right) ; i^{(1)}=-\frac{1}{3} i_{b} ; i^{(2)}=-\frac{1}{3} i_{c}$ and $i^{(3)}=\frac{1}{3}\left(i_{b}-i_{c}\right)$.

Similarly, the conditions in modal domain for other faults were obtained. Four fault parameters viz. Wmm0, Wmm1, $W m m 2$ and $W m m 3$ are defined and obtained using wavelet analysis as follows. ' $W m m 0$ ' is the first modulus maximum of mode- 0 traveling wave which corresponds to the first 
strike of mode-0 component wave at relay location. Similarly, 'Wmm1', 'Wmm2' and ' $W m m 3$ ' are the first modulus maxima of mode-1, mode- 2 and mode- 3 waves launched by the fault, respectively. From the conditions in modal domain, the rules using fault parameters to select the faulted phase can be written as follows. The fault parameters separately or in combination are compared with threshold values $\varepsilon_{1,} \varepsilon_{2}$, or $\varepsilon_{3}$, whose values were obtained using simulation studies. The rules are:

1. If $|W m m 0|>\varepsilon_{1}$ and $|W m m 3|<\varepsilon_{2}$ and

$\| W m m 1|-| W m m 2||<\varepsilon_{2}$, then fault is AG.

2. If $|W m m 0|>\varepsilon_{1}$ and $|W m m 2|<\varepsilon_{2}$ and

||$W m m 1|-| W m m 3||<\varepsilon_{2}$, then fault is BG.

3. If $|W m m 0|>\varepsilon_{1}$ and $|W m m 1|<\varepsilon_{2}$ and

||$W m m 2|-| W m m 3||<\varepsilon_{2}$, then fault is CG.

4. If $|W m m 0|>\varepsilon_{1}$ and $\| W m m 2+W m m 3|-| W m m 0||<\varepsilon_{3}$, and Polarity $(W m m 2+W m m 3)=$ Polarity $(W m m 0)$, then fault is $\mathrm{ABG}$.

5. If $|W m m 0|>\varepsilon_{1}$ and $\| W m m 1+W m m 2|-| W m m 0||<\varepsilon_{3}$, and Polarity $(W m m 1+W m m 2) \neq$ Polarity $(W m m 0)$, then fault is BCG.

6. If $|W m m 0|>\varepsilon_{1}$ and $\| W m m 1-W m m 3|-| W m m 0||<\varepsilon_{3}$, and Polarity $(W m m 1-W m m 3)=$ Polarity $(W m m 0)$, then fault is ACG.

7. If $|W m m 0|<\varepsilon_{1}$ and $|W m m 2|-|W m m 3|<\varepsilon_{2}$ and

$|W m m 2|+|W m m 3|-|W m m 1| \mid<\varepsilon_{2}$ then fault is AB.

8. If $|W m m 0|<\varepsilon_{1}$ and $|W m m 1|-|W m m 2|<\varepsilon_{2}$ and

$|W m m 1|+|W m m 2|-|W m m 3| \mid<\varepsilon_{2}$ then fault is BC.

9. If $|W m m 0|<\varepsilon_{1}$ and $|W m m 1|-|W m m 3|<\varepsilon_{2}$ and

$|W m m 1|+|W m m 3|-|W m m 2| \mid<\varepsilon_{2}$ then fault is AC.

\section{STUDY SYSTEM}

The Sim Power System which is an extension to the Simulink of MATLAB software was used to simulate the double end fed power system [14]. The $200 \mathrm{~km}, 400 \mathrm{KV}$ transmission line as shown in Fig. 1 was modeled using distributed parameter model.

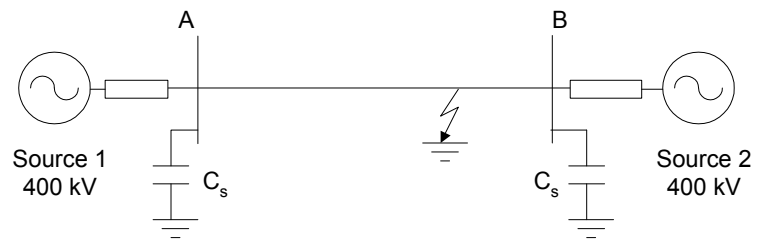

Fig. 1 Study system

The value of bus bar capacitance $\mathrm{C}_{\mathrm{s}}$ is assumed to be 0.1 $\mu \mathrm{F}$. The network and line data is given below.

Transmission line data:
Positive sequence impedance, $Z_{1}=5.5+\mathrm{j} 63.0 \Omega$

Zero sequence impedance, $Z_{0}=55.0+\mathrm{j} 205.3 \Omega$

Positive sequence susceptance, $B_{1}=4.084 \mu \mathrm{S} / \mathrm{km}$

Zero sequence susceptance, $B_{0}=2.67 \mu \mathrm{S} / \mathrm{km}$

Equivalent system at both terminals:

Positive sequence impedance $=1.31+\mathrm{j} 15.0 \Omega$

Negative sequence impedance $=2.33+\mathrm{j} 26.6 \Omega$

\section{Algorithm}

Since the transient based techniques are based on the extraction of the characteristics of transient components generated by fault, a high sampling rate is always set to capture the wave fronts of transients. The sampling period is chosen to be $1 \mu \mathrm{s}$. The algorithm to classify the fault and hence select the faulted phase is presented below.

1. Obtain the superimposed current signals $\Delta \mathrm{i}_{\mathrm{p}}(\mathrm{t})$ from the transducer output. $(p=a, b, c)$

2. Transform the time domain signals into modal domain using Karrenbauer transformation matrix.

3. Analyze the modal components using wavelet transform and obtain the four fault parameters.

4. Differentiate between the faults as those involving ground (LG and LLG faults) and those which do not involve ground (LL and LLL faults), based on comparison of fault parameter $W m m 0$ with the threshold value $\varepsilon_{1}$.

5. If fault involves ground, differential between LG and LLG faults and then identify individual fault based on the rules given in the fault parameterization subsection.

6. If fault does not involve ground, identify individual LL fault based on the rules given in the fault parameterization subsection else fault is three phase fault.

\section{RESUltS AND DisCUSSION}

Simulation studies were carried out on the typical model of $400 \mathrm{kV}$ transmission line systems shown in Fig. 1. A variety of fault scenarios, including different fault inception angles, fault resistances, fault locations and fault types, had been simulated to evaluate the validity of this approach. All ten types of faults involving single phase, two phases and three phases were evaluated and sample results are given in Table I. These examples demonstrate the validity of faulted phase selection scheme. The scheme had also been tested on many other faults, and the results were found to be satisfactory as those presented here.

TABLE I: TEST RESULTS FOR FAULTED PHASE SELECTOR

\begin{tabular}{|c|c|c|c|c|c|c|c|}
\hline \multirow{2}{*}{$\begin{array}{l}\text { Fault } \\
\text { Location } \\
(\mathrm{km})\end{array}$} & \multirow{2}{*}{$\begin{array}{l}\text { Fault } \\
\text { inception } \\
\text { time (s) }\end{array}$} & \multirow{2}{*}{$\begin{array}{l}\text { Fault } \\
\text { resistance } \\
(\Omega)\end{array}$} & \multicolumn{4}{|c|}{ Fault parameters } & \multirow{2}{*}{$\begin{array}{l}\text { Fault } \\
\text { type }\end{array}$} \\
\hline & & & Wmm0 & Wmml & $W m m 2$ & Wmm3 & \\
\hline 79 & 0.0405 & 41 & -309.7 & 308 & - & -308 & $\mathrm{BG}$ \\
\hline 123 & 0.0417 & 89 & 101.4 & - & -102 & -102 & $\mathrm{CG}$ \\
\hline 150 & 0.0408 & 100 & 84.51 & 86.92 & 86.92 & - & $\mathrm{AG}$ \\
\hline 110 & 0.0434 & 72 & 27.03 & 916.9 & 471.9 & -445 & $\mathrm{ABG}$ \\
\hline 50 & 0.0423 & 100 & -131.3 & 355.9 & -224.6 & -580.5 & $\mathrm{BCG}$ \\
\hline 172 & 0.0391 & 124 & 109.3 & -257.4 & -629.3 & -371.9 & $\mathrm{ACG}$ \\
\hline 16 & 0.0431 & 10 & - & 888.5 & 444.3 & -444.3 & $\mathrm{AB}$ \\
\hline 189 & 0.0442 & 8 & - & 78.99 & -78.95 & -157.9 & $\mathrm{BC}$ \\
\hline 78 & 0.0403 & 9 & - & -126.1 & -252.2 & -126.1 & $\mathrm{AC}$ \\
\hline 37 & 0.0425 & 7 & - & 902.8 & 380.6 & -522.2 & $\mathrm{ABC}$ \\
\hline
\end{tabular}


The waveforms obtained for BG fault with fault conditions corresponding to the first row of Table I, are given in Fig.2. Fig. 2 (a) depicts the superimposed currents $\Delta \mathrm{i}_{\mathrm{abc}}(\mathrm{t})$, which contain all the essential information regarding the traveling wave components. The superimposed signals can be separated from the pre-fault components during the first cycle of fault duration by subtracting from the real time signal values the delayed pre fault signal values in the previous cycle. Fig. 2 (b), (c) and (d) typify the mode-0, mode-1, mode-2 and mode-3 components of the superimposed currents respectively. X-axis labels of Fig.2, 'index' refers to the sample numbers.

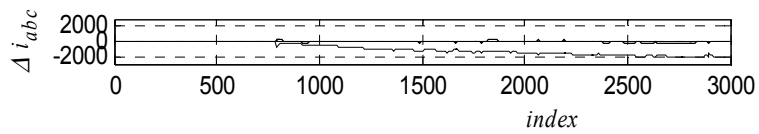

(a)

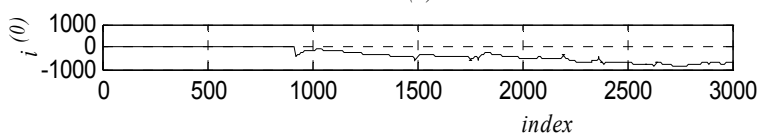

(b)

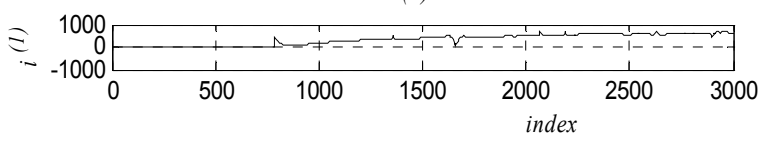

(c)

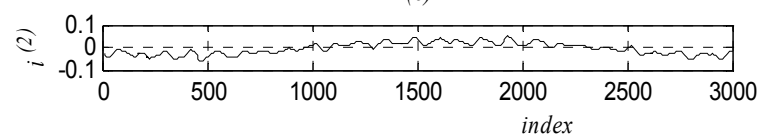

(d)

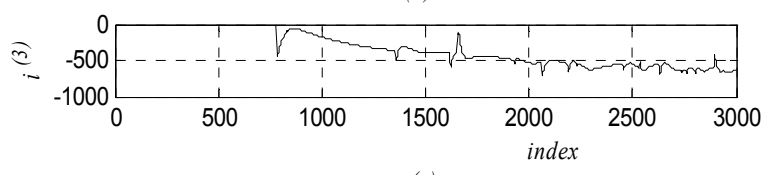

(e)

Fig. 2 (a) Superimposed currents and their (b) mode-0 (c) mode-1 (d) mode-2 and (e) mode-3 components for BG fault.

The wavelet analysis is done on all the four modal signals viz. $i^{(0)}, i^{(1)}, i^{(2)}$ and $i^{(3)}$. The modulus maxima of these signals were obtained using functions in WaveLab toolbox. These are shown in Fig. 3. The Y-labels of Fig. 3, WMM k refer to the wavelet modulus maxima for the corresponding mode-k signal, where $\mathrm{k}$ takes the values $0,1,2$ and 3 . The first modulus maximum in each of the WMM signal corresponds to the first strike of modal traveling wave at the bus. The magnitudes and polarities of first modulus maxima are used in the algorithm. The values of fault parameters obtained from Fig. 3 are given in first row of Table I and using the algorithm, the faulted phase was correctly identified.

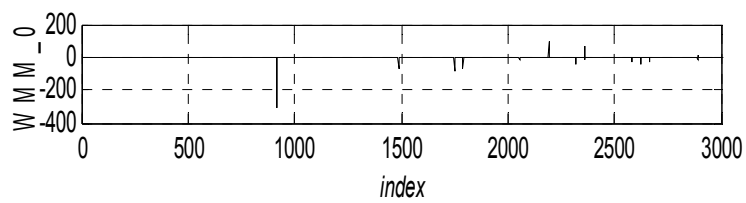

(a)

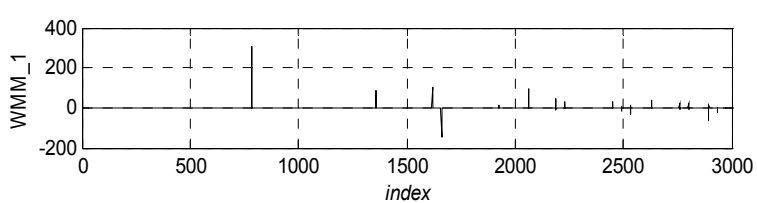

(b)

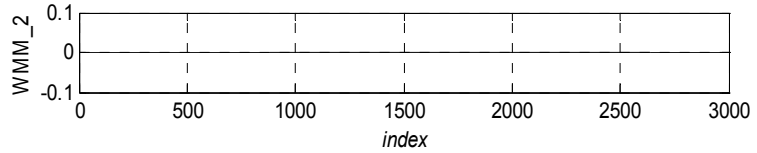

(c)

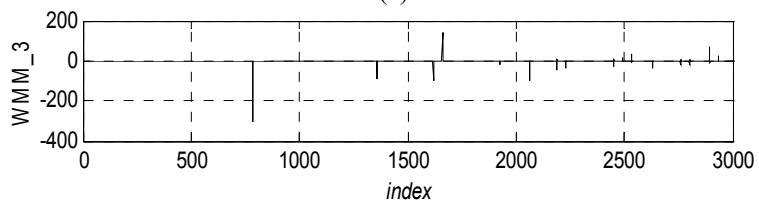

(d)

Fig. 3 Wavelet modulus maxima of (a) mode -0 (b) mode- 1 (c) mode-2 and (d) mode- 3 components of currents for BG fault.

\section{A. Effect of fault resistance}

The effect of fault resistance on the selection of faulted phase was considered in the paper by varying it up to $300 \Omega$. The sample results for AG and BCG faults for high values of fault resistances, occurring at a distance of $150 \mathrm{~km}$ from bus 'A' are shown in Table II. The values of fault discriminating parameters are slightly affected; however the conditions for the selection of faulted phase are still valid for the respective faults and hence there is correct identification of faults.

\begin{tabular}{|c|c|c|c|c|c|}
\hline \multicolumn{5}{|c|}{ TABLE II: EFFECT OF FAULT RESISTANCE } & $\begin{array}{l}\text { Fault } \\
\text { type } \\
\begin{array}{c}\text { Fault } \\
\text { resistance } \\
(\Omega)\end{array}\end{array}$ \\
\cline { 2 - 5 } & $W m m 0$ & $W m m 1$ & $W m m 2$ & $W m m 2$ & \\
\hline 200 & 63.32 & 65.38 & 65.38 & - & AG \\
\hline 300 & 50.85 & 51.47 & 51.47 & - & AG \\
\hline 200 & -82.35 & -278.4 & 362.9 & -641.3 & BCG \\
\hline 300 & -63.29 & 363.2 & -288.2 & -641.4 & BCG \\
\hline
\end{tabular}

\section{B. Effect of fault inception angle}

For the cases of fault inception at zero crossing, theoretically, no traveling wave is generated from the fault location. Therefore, traveling wave based faulted phase selector cannot operate under this situation. But as long as fault does not occur at absolute zero, the faulted phase selector algorithm based on traveling wave can work properly. The fault inception angle for a particular LG fault is varied up to $90^{\circ}$. The simulation results given in table III, show that the proposed algorithm works correctly even with a low inception angle of $5^{\circ}$. In practice, the insulation breakdown, which results into a fault, is always caused by a significant voltage.

\begin{tabular}{|c|c|c|c|c|c|}
\hline \multirow{2}{*}{$\begin{array}{l}\text { Fault } \\
\text { inception } \\
\text { angle } \\
\text { (degree) }\end{array}$} & \multicolumn{4}{|c|}{ Fault parameters } & \multirow{2}{*}{$\begin{array}{l}\text { Fault } \\
\text { type }\end{array}$} \\
\hline & Wmm0 & $W m m 1$ & Wmm2 & Wmm2 & \\
\hline 5 & 11.65 & 11.73 & 11.72 & - & $\mathrm{AG}$ \\
\hline 45 & 172 & 170.3 & 170.3 & - & $\mathrm{AG}$ \\
\hline 90 & 256.3 & 252.9 & 252.9 & - & $\mathrm{AG}$ \\
\hline
\end{tabular}




\section{CONCLUSION}

The paper presented a high speed faulted phase selection approach, based on the wavelet analysis of fault induced current traveling waves for use in the application of single pole reclosing on EHV transmission lines. In this approach, wavelet modulus maxima technique was used to capture the high frequency traveling waves in modal domain. The results showed that faulted phase selection can be performed within a quarter of cycle and also it is insensitive to uncontrolled parameters like fault location, fault resistance, fault inception angle.

\section{REFERENCES}

[1] Y.G.Paithankar, S.R.Bhide, Fundamentals of Power System Protection, Prentice-Hall of India, 2010.

[2] IEEE/PSRC Report, "Single Phase Tripping and Auto Reclosing of Transmission Lines," IEEE Trans. on Power Delivery, vol 7, no. 1, Jan.1992, pp. 182-192.

[3] T. Dalstein, B. Kulicke, "Neural network approach to fault classification for high speed protective relaying," IEEE Transactions on Power Delivery, vol. 10, no. 2, April 1995, pp. 1002-1009.

[4] B. Das, and J. V. Reddy, "Fuzzy-logic-based fault classification scheme for digital distance protection," IEEE Transactions on Power Delivery, vol. 20, no. 2, April 2005, pp. 609 -616.

[5] B. Ravikumar, D. Thukaram and H.P. Khincha, "Application of support vector machines for fault diagnosis in power transmission system," IET Gener. Transm. Distrib., 2008, 2, (1), pp. 119-130.

[6] S. El Safty, A. El-ZonkolyInt., "Applying wavelet entropy principle in fault classification," Journal of Electrical Power and Energy Systems, vol. 31, Issue 10, November-December 2009, pp. 604-607.

[7] K. M. Silva, B. A. Souza, and N. S. D. Brito, "Fault detection and classification in transmission lines lased on wavelet transform and ANN," IEEE Trans. on Power Delivery, vol. 21, no. 4, October 2006, pp. $2058-2063$.

[8] V.S.Kale, S.R.Bhide, P.P.Bedekar, Faulted Phase Selection on Double Circuit Transmission Line using Wavelet Transform and Neural Network," IEEE International Conference on Power Systems, 2009, pp. $1-6$.
[9] A.A.Hajjar, M.M.Mansour, H.A.Talaat, "High phase order power transmission lines relaying approach based on the wavelet analysis of the fault generated traveling waves," IEEE Int. Universities Power Engg. Conf. 2004, vol. 2, pp. 805-809.

[10] X.G.Wang, S.F.Huang, Q.K.Liu, "A fault classification method based on spectrum of fault component for EHV lines," IEEE Power \& Energy Society general meeting, 2008, pp. 1-6.

[11] P.Kumar, M.Jamil, M.S.Thomas, Moinuddin, "Fuzzy approach to fault classification for transmission line protection," Proc. IEEE TENCON 99 Conf., vol. 2, pp. 1046-1050.

[12] S. Qianli, Dong Xinzhou, "New Approach of fault detection and fault phase selection based on initial current traveling waves," IEEE Power Engg. Society Summer Meeting, 2002, vol. 1, pp. 393-397.

[13] Kim C.H., Aggarwal, R., "Wavelet transforms in power systems," IET Power Engineering Journal, vol. 15, Aug. 2001, pp. 193-200.

[14] MATLAB 7.1 User's Guide for Simulink, SimPowerSystem.

V. S. Kale is working as Assistant Professor in the Department of Electrical Engineering, Visvesvaraya National Institute of Technology, Nagpur, India. His research interests include application of wavelet transform and $\mathrm{AI}$ techniques to power system protection.

S. R. Bhide received B.E., M.Tech and Ph.D. degrees from Nagpur University, Nagpur (India). $\mathrm{He}$ is an Associate Professor in the Department of Electrical Engineering, Visvesvaraya National Institute of Technology, Nagpur, India. He has co-authored a book titled "Fundamentals of Power System Protection", published by Prentice Hall of India. His special area of interest includes application of AI techniques to power system protection.

P. P. Bedekar is currently pursuing the Ph.D. degree in the Department of Electrical Engineering, Visvesvaraya National Institute of Technology, Nagpur, India. His research interests include power system protection, optimization, and AI techniques. 